\title{
Modal Decoupled Dynamics Feed-Forward Active Force Control of Spatial Multi-DOF Parallel Robotic Manipulator
}

\author{
Xinjian Niu, ${ }^{1}$ Chifu Yang $\left(\mathbb{D},{ }^{1}\right.$ Bowen Tian, ${ }^{2}$ Xiang Li $\mathbb{D},{ }^{3}$ and Junwei Han ${ }^{1}$ \\ ${ }^{1}$ State Key Laboratory of Robotics and System, Harbin Institute of Technology, Harbin 150001, China \\ ${ }^{2}$ Department of Economic and Trade, School of Business Administration, Zhongnan University of Economics and Law, \\ Wuhan 430000, China \\ ${ }^{3}$ Department of Mechatronics Engineering, Harbin Institute of Technology, Harbin 150001, China
}

Correspondence should be addressed to Xiang Li; lxiang0818@163.com

Received 10 June 2018; Revised 16 August 2018; Accepted 19 September 2018; Published 16 April 2019

Guest Editor: Carlos Llopis-Albert

Copyright (C) 2019 Xinjian Niu et al. This is an open access article distributed under the Creative Commons Attribution License, which permits unrestricted use, distribution, and reproduction in any medium, provided the original work is properly cited.

According to the parallel mechanism theory, this paper proposes a novel intelligent robotic spine brace for the treatment of scoliosis. Nevertheless, this type of parallel mechanism has the following disadvantages: strong dynamic coupling in task space or joint space, adverse effect of system's gravity, and lower response frequency in roll and pitch orientations, which seriously affect the performance of the system. In order to solve those boring problems, this paper presents a novel active force control structure, modal space dynamic feed-forward (MSDF) force control strategy. Besides, this paper expresses the intelligent robotic brace system model including the dynamic and kinematic models and the electric actuator model with Kane strategy. The stability of the intelligent system with the novel control strategy is proved. In order to evaluate the performance of the presented MSDF force control method, this paper builds the parallel mechanism experimental platform. It can be seen from experimental results that the proposed motion control method solves these boring problems well.

\section{Instruction}

Scoliosis is a three-dimensional (3D) abnormal curvature of the spine [1]. However, current braces are mainly used to treat the lateral curvature of the spine, which are little effective for the anterior and posterior curvature of the spine [2-5]. Besides, since corrective forces are applied on human spine passively using current braces, it is difficult to control the forces accurately, which brings difficulties in the treatment of the scoliosis. A new intelligent parallel manipulator system is designed in this paper for exerting $3 \mathrm{D}$ active corrective forces on the spine of patients [6]. A spatial multi-degree of freedom (multi-DOF) parallel robotic manipulator actuated by electrical actuators is used as the robotic brace in this paper, since this parallel structure has high precision, high reliability, and high power weight radio and offers $3 \mathrm{D}$ active corrective forces [7-9]. However, this type of parallel mechanism has the following disadvantages, strong dynamic coupling in task space or joint space, adverse effect of system's gravity and lower response frequency in roll and pitch orientations, which seriously affect the performance of the system. Because of the above boring problems of the parallel mechanism, it is difficult to improve the performance of the system just with the typical proportion-integral-derivative (PID) control method in traditional physical space [10-12]. Fortunately, the dynamic feed-forward control strategy in modal space can solve these problems excellently.

A lot of studies have reported this parallel mechanism widely [13-16], and this structure was used in many areas practically $[17,18]$. For solving those inherent properties, a lot of control methods have been presented for parallel structure [19-21]. Those control strategies were all presented in traditional physical space, joint space, or task space [22-25]. Without taking into account the system dynamics and system kinematics, a series control strategy with the disturbance observer for parallel mechanism was designed in joint space [26]. This study increases the anti-interference characteristics of the system, which improves the performance of the system. However, they ignored the influence of the gravity term, which is important for the system. Yang et al. developed 
a proportion-derivative (PD) control strategy with gravity compensation for the hydraulic 6-DOF parallel manipulator to solve the problem of the device's gravity, which improve the track tracking performance well [27]. However, they did not consider coupling problem of the parallel mechanism in all six directions, which infected tracking precision serious. Based on the observer, a backstepping control algorithm for forward kinematics solver is studied taking into account the actuator's friction [28]. In order to improve the performance of the system, the friction of the actuator is considered in this literature. Nevertheless, the gravity of the system was also ignored in this study, which had bad effects on the parallel system. In previous literatures only the problem of tracking motion trajectories using a variety of basic and advanced control methods is considered. However, it is critical for most parallel robotic applications to detect contact force between robot and environment, for example, assembly robots, grinding robots, surgery robots, and rehabilitation robots which involve extensive contact with environment. The development of force control strategy for parallel robotic manipulators is later than that of motion control. Noshadi et al. presented an active force control strategy combined with the classical PID controller for a 3-RRR (revolute-revoluterevolute) 3-DOF planar parallel robotic manipulator [29]. Bera et al. proposed a virtual foundations force control in parallel robotic manipulator using bond graph modeling [30]. Lopes et al. reported a method to obtain high performance of active force control by combing a robotic controlled impedance device (RCID) with a commercial industrial robot [31]. Goertzen et al. proposed a new application of a velocitybased force control method used for robotic biomechanical testing [32]. Wen et al. presented a fuzzy identification strategy for achieving the system model of the multi-DOF spatial redundantly actuated parallel robot [33]. Since those inherent shortcomings of the parallel mechanism, the performance of parallel robotic manipulator cannot be achieved satisfactorily using the above-mentioned force control strategies in joint space and work space. For solving those inherent properties, a decoupling motion control method is studied in modal space $[34,35]$. Nevertheless, this control structure is designed just for motion control of parallel robotic manipulator. Yang et al. proposed a novel modal space decouple control method for force trajectory tracking, which solved the dynamic coupling in force field [36]. However, this novel control structure is developed based on the classical PID control strategy, which decoupled the dynamic coupling, but neglected gravity term of the robot system. Thus, the current force control strategies for the parallel mechanism are all controlled in joint space or workspace which could not solve the dynamic coupling problem of the parallel manipulator. Besides, most current control methods ignored the gravity term of the device which is critical for achieving high-precision corrective forces. This paper develops a novel force control strategy, which solves those boring inherent properties of the parallel system.

The main contribution of this article is to design a new control structure, modal space dynamic feed-forward (MSDF) active force control for parallel robotic manipulators which can eliminate the bad effects of those inherit properties such as dynamic coupling, low response frequency, and

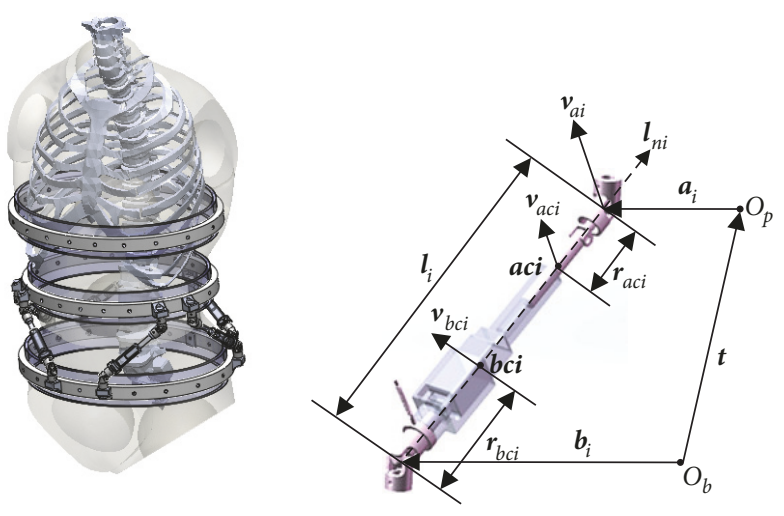

FIgURE 1: Structure of the novel brace.

gravity term in six directions. In comparison to those control structures in traditional physical space, the proposed control strategy improves performances of parallel mechanism effectively.

\section{System Model}

The intelligent robotic system consists of two Stewart platforms in series. As can be seen in Figure 1, this intelligent system is driven with twelve actuators and there is a kinematic chain of universal prismatic spherical (UPS) in each leg. Since the upper platform has the same structure with the lower platform, this paper just describes the system model of the lower platform.

2.1. Dynamics Model. When using the rigid body modeling theory and establishing the dynamic model of the parallel robotic manipulator system, there are the following assumptions: (1) the moving platform and fixed platform are both rigid bodies. (2) The piston rod and cylinder are both rigid bodies. (3) Hinges and other connectors are all rigid connectors with negligible masses. According to above assumptions, the system consists of 13 rigid bodies including 6 piston rods, 6 cylinders, and one moving platform, respectively. Based on the Newton's second law and angular momentum theory, inertia force and inertia moment of the system are expressed as

$$
\begin{aligned}
\boldsymbol{F}_{p} & =\boldsymbol{m}_{p} \ddot{\boldsymbol{t}} \\
\boldsymbol{M}_{p} & =\boldsymbol{I}_{p} \dot{\boldsymbol{\omega}}^{L}+\boldsymbol{\omega}^{L} \times \boldsymbol{I}_{p} \boldsymbol{\omega}^{L}
\end{aligned}
$$

in which $\boldsymbol{F}_{p}$ is the inertia force. $\boldsymbol{M}_{p}$ is the inertia moment and $\boldsymbol{m}_{p}$ means the mass of the motion upper platform. $\boldsymbol{t}$ is the linear vector of the body framework relative to the fixed framework. $\omega^{L}$ is the upper platform's angular velocity vector in fixed framework. $\boldsymbol{I}_{p}$ means the inertia tensor in inertial framework and can be expressed as

$$
\boldsymbol{I}_{p}=\boldsymbol{R} \boldsymbol{I}_{p}^{p} \boldsymbol{R}^{T}
$$


in which $\boldsymbol{I}_{p}^{p}$ means inertia tensor in body framework. $\boldsymbol{R}$ denotes the transformation matrix from the body framework to the inertia framework and $\boldsymbol{R}^{T}$ is the transpose of $\boldsymbol{R}$.

Based on (1) and (2), the generalized inertia force of motion platform is expressed as

$$
\begin{aligned}
\boldsymbol{F}_{p}{ }^{*} & =-\boldsymbol{M}_{\boldsymbol{p}} \ddot{\overline{\boldsymbol{q}}}-\boldsymbol{C}_{\boldsymbol{p}} \dot{\bar{q}} \\
& =\left(\begin{array}{cc}
\boldsymbol{m}_{\boldsymbol{p}} \boldsymbol{I} & 0 \\
0 & \boldsymbol{I}_{\boldsymbol{p}}
\end{array}\right)\left[\begin{array}{c}
\ddot{\boldsymbol{t}} \\
\dot{\omega}^{L}
\end{array}\right]-\left(\begin{array}{cc}
0_{3 \times 3} & 0 \\
0_{3 \times 3} & \widetilde{\boldsymbol{\omega}}^{L} \boldsymbol{I}_{\boldsymbol{p}}
\end{array}\right)\left[\begin{array}{c}
\dot{\boldsymbol{t}} \\
\omega^{L}
\end{array}\right]
\end{aligned}
$$

in which $\widetilde{\boldsymbol{\omega}}^{L}$ means the angular velocity's skew symmetric matrix in inertia framework. $\dot{\overline{\boldsymbol{q}}} \ddot{\overline{\boldsymbol{q}}}$ are the generalized velocity and acceleration in the inertia framework. $\boldsymbol{C}_{\boldsymbol{p}}$ is the damping coefficient of the system.

In the same way, according to the angular momentum theorem and Newton's second law, inertia moment force and moment of piston rod is described as

$$
\begin{aligned}
\boldsymbol{F}_{r v i} & =\boldsymbol{m}_{a} \dot{\boldsymbol{v}}_{a c i} \\
\boldsymbol{M}_{r w i} & =\boldsymbol{I}_{a i} \dot{\boldsymbol{\omega}}_{l i}+\boldsymbol{\omega}_{l i} \times \boldsymbol{I}_{a i} \boldsymbol{\omega}_{l i}
\end{aligned}
$$

where $\boldsymbol{F}_{r v i}, \boldsymbol{M}_{r w i}$ are inertia force and inertia moment of piston rod. $\boldsymbol{m}_{a}$ means the mass of piston rod. $\boldsymbol{v}_{a c i}$ denotes piston rod's centroid velocity. $\omega_{l i}$ means the leg's angular velocity. $\boldsymbol{I}_{a i}$ denotes the piston rod's inertia tensor.

The leg's angular velocity and the piston rod's centroid velocity are expressed with the motion platform's generalized velocity.

$$
\begin{aligned}
\boldsymbol{v}_{a c i} & =\boldsymbol{J}_{a c i, a i} \boldsymbol{J}_{a i, \bar{q}} \dot{\bar{q}} \\
\boldsymbol{\omega}_{l i} & =\boldsymbol{J}_{w i, a i} \boldsymbol{J}_{a i, \overline{\bar{q}}} \dot{\bar{q}}
\end{aligned}
$$

where $\boldsymbol{J}_{a c i, a i}$ means the Jacobi matrix from the velocity of upper hinge point to the piston rod's centroid velocity. $\boldsymbol{J}_{a i, \bar{q}}$ denotes the Jacobi matrix from the motion platform's generalized velocity to the velocity of upper hinge point. $\boldsymbol{J}_{w i, a i}$ is the Jacobi matrix from the velocity of upper hinge point to the leg's angular velocity.

Based on (5), (6), (7), and (8), the piston rod's generalized inertia force in fixed framework is gotten

$$
\begin{aligned}
\boldsymbol{F}_{r i}^{*} & =\boldsymbol{J}_{a i, \bar{q}}^{T} \boldsymbol{J}_{a c i, a i}^{T} \boldsymbol{F}_{r v i}+\boldsymbol{J}_{a i, \bar{q}}^{T} \boldsymbol{J}_{w i, a i}^{T} \boldsymbol{M}_{r w i} \\
& =\boldsymbol{J}_{a i, \bar{q}}^{T}\left(\boldsymbol{J}_{a c i, a i}^{T} \boldsymbol{m}_{a} \boldsymbol{J}_{a c i, a i}+\boldsymbol{J}_{w i, a i}^{T} \boldsymbol{I}_{a i} \boldsymbol{J}_{w i, a i}\right) \boldsymbol{J}_{a i, \bar{q}} \ddot{\bar{q}} \\
& +\left\{\boldsymbol{J}_{a i, \bar{q}}^{T}\left[\frac{\boldsymbol{J}_{a c i, a i}^{T} \boldsymbol{m}_{a} d\left(\boldsymbol{J}_{a c i, a i} \boldsymbol{J}_{a i, \bar{q}}\right)}{d t}\right]\right. \\
& +\boldsymbol{J}_{a i, \bar{q}}^{T} \boldsymbol{J}_{w i, a i}^{T}\left[\frac{\boldsymbol{I}_{a i} d\left(\boldsymbol{J}_{w i, a i} \boldsymbol{J}_{a i, \bar{q}}\right)}{d t}+\boldsymbol{J}_{w i, a i} \boldsymbol{J}_{a i, \bar{q}} \dot{\bar{q}}\right. \\
& \left.\left.\times \boldsymbol{I}_{a i} \boldsymbol{J}_{w i, a i} \boldsymbol{J}_{a i, \bar{q}}\right]\right\} \dot{\overline{\boldsymbol{q}}}
\end{aligned}
$$

where $\boldsymbol{F}_{r i}^{*}$ is the generalized inertia force of piston rod in the inertial coordinate framework. $\boldsymbol{J}_{a c i, a i}^{T}, \boldsymbol{J}_{a i, \bar{q}}^{T}, \boldsymbol{J}_{w i, a i}^{T}$ are the transpose of matrix $\boldsymbol{J}_{a c i, a i}, \boldsymbol{J}_{a i, \bar{q}}, \boldsymbol{J}_{w i, a i}$.
In the same way, the cylinder's generalized force in fixed framework is expressed as

$$
\begin{aligned}
\boldsymbol{F}_{c i}^{*} & =\boldsymbol{J}_{a i, \bar{q}}^{T} \boldsymbol{J}_{b c i, a i}^{T} \boldsymbol{F}_{c v i}+\boldsymbol{J}_{a i, \bar{q}}^{T} \boldsymbol{J}_{w i, a i}^{T} \boldsymbol{M}_{c w i} \\
& =\boldsymbol{J}_{a i, \bar{q}}^{T}\left(\boldsymbol{J}_{b c i, a i}^{T} \boldsymbol{m}_{b} \boldsymbol{J}_{b c i, a i}+\boldsymbol{J}_{w i, a i}^{T} \boldsymbol{I}_{b i} \boldsymbol{J}_{w i, a i}\right) \boldsymbol{J}_{a i, \overline{\bar{q}}} \ddot{\overline{\boldsymbol{q}}} \\
& +\left\{\boldsymbol{J}_{a i, \bar{q}}^{T}\left[\frac{\boldsymbol{J}_{b c i, a i}^{T} \boldsymbol{m}_{b} d\left(\boldsymbol{J}_{b c i, a i} \boldsymbol{J}_{a i, \bar{q}}\right)}{d t}\right]\right. \\
& +\boldsymbol{J}_{a i, \bar{q}}^{T} \boldsymbol{J}_{w i, a i}^{T}\left[\frac{\boldsymbol{I}_{b i} d\left(\boldsymbol{J}_{w i, a i} \boldsymbol{J}_{a i, \bar{q}}\right)}{d t}+\boldsymbol{J}_{w i, a i} \boldsymbol{J}_{a i, \bar{q}} \dot{\bar{q}}\right. \\
& \left.\left.\times \boldsymbol{I}_{b i} \boldsymbol{J}_{w i, a i} \boldsymbol{J}_{a i, \bar{q}}\right]\right\} \dot{\overline{\boldsymbol{q}}}
\end{aligned}
$$

where $\boldsymbol{F}_{c i}^{*}$ is the generalized inertia force of the cylinder. $\boldsymbol{m}_{b}$ is the cylinder's mass. $\boldsymbol{I}_{b i}$ is the cylinder's inertia tensor. $\boldsymbol{J}_{b c i, a i}$ means the Jacobi matrix from the velocity of upper hinge point to the cylinder's centroid velocity. $\boldsymbol{J}_{b c i, a i}^{T}$ is the transpose of matrix. $\boldsymbol{F}_{c v i}, \boldsymbol{M}_{c w i}$ are inertia force and inertia moment of the cylinder.

According to Kane approach and based on (4), (9), and (10), the dynamic model of the intelligent robotic system is described as

$$
\boldsymbol{\tau}-\boldsymbol{F}_{c}+\boldsymbol{F}_{p}^{*}+\sum_{i=1}^{6} \boldsymbol{F}_{r i}^{*}+\sum_{i=1}^{6} \boldsymbol{F}_{c i}^{*}+\boldsymbol{G}^{*}=0
$$

in which $\boldsymbol{F}_{c}$ means the contact force. $\boldsymbol{G}^{*}$ is the device's gravity. $\boldsymbol{\tau}$ denotes the generalized force and is expressed as

$$
\boldsymbol{\tau}=\boldsymbol{J}_{l \bar{q}}^{T} \boldsymbol{F}_{a}
$$

in which $\boldsymbol{J}_{l \bar{q}}^{T}$ denotes the Jacobi matrix from the generalized velocity to the leg's velocity. $\boldsymbol{F}_{a}$ means the net output force of the actuator.

Base on (11), the standard formula of the system model in task space is written as

$$
\boldsymbol{M}(\bar{q}) \ddot{\bar{q}}+\boldsymbol{N}(\bar{q}, \dot{\bar{q}}) \dot{\bar{q}}+\boldsymbol{G}(\bar{q})+\boldsymbol{F}_{c}=\boldsymbol{\tau}
$$

in which $\boldsymbol{M}$ is the system's mass matrix. $\boldsymbol{N}$ means the centrifugal term. $\boldsymbol{G}$ denotes the device's gravity term. $\bar{q}$ means the motion platform's generalized pose.

In addition (13) is rewritten in joint space

$$
\boldsymbol{M}_{l}(l) \ddot{l}+\boldsymbol{C}_{l}(l, \dot{l}) \dot{l}+\boldsymbol{G}_{l}(l)+\boldsymbol{F}_{c l}=\boldsymbol{F}_{a}
$$




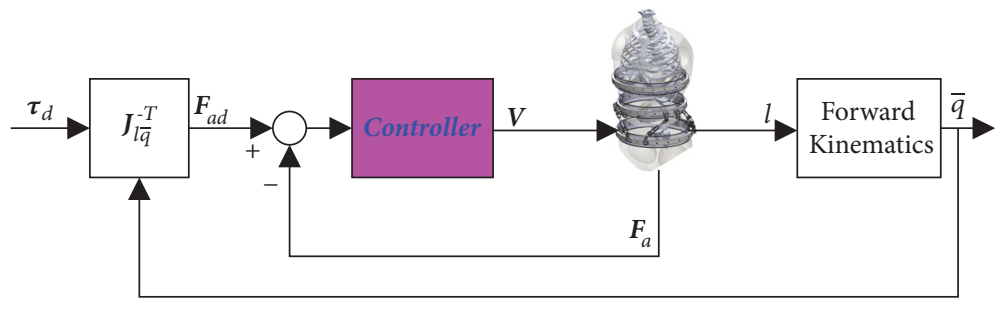

FIgURE 2: Active force control strategy in traditional physical space.

in which $\boldsymbol{M}_{l}$ means mass matrix. $\boldsymbol{C}_{l}$ denotes centrifugal force. $\boldsymbol{G}_{l}$ shows the gravity term. With the Jacobi matrix $\boldsymbol{J}_{\overline{l q}}$, the work space and the joint space are connected as follows:

$$
\begin{aligned}
\dot{l} & =\boldsymbol{J}_{l \bar{q}} \dot{\bar{q}} \\
\boldsymbol{M}_{l} & =\boldsymbol{J}_{l \bar{q}}^{-T} \boldsymbol{M} \boldsymbol{J}_{\overline{l q}}^{-1} \\
\boldsymbol{C}_{l} & =\boldsymbol{J}_{l \bar{q}}^{-T}\left(\boldsymbol{M} \dot{J}_{\bar{q}}^{-1}+\boldsymbol{N J}_{l \bar{q}}^{-1}\right) \\
\boldsymbol{F}_{c l} & =\boldsymbol{J}_{l \bar{q}}^{-T} \boldsymbol{F}_{c} \\
\boldsymbol{G}_{l} & =\boldsymbol{J}_{l \bar{q}}^{-T} \boldsymbol{G}
\end{aligned}
$$

In joint space, the system's mass matrix is a real symmetric and positive definite matrix [37].

2.2. Kinematics Model. Current studies have developed the kinematics of parallel mechanism widely [38, 39]. Hence, this paper just describes the kinematics briefly. According to Figure 1, the inverse kinematics is described with the geometric approach

$$
l_{i}=\sqrt{\boldsymbol{l}_{i}^{T} \boldsymbol{l}_{i}}=\sqrt{\left(\boldsymbol{t}+\boldsymbol{R} \boldsymbol{a}_{i}^{p}-\boldsymbol{b}_{i}\right)^{T}\left(\boldsymbol{t}+\boldsymbol{R a}_{i}^{p}-\boldsymbol{b}_{i}\right)}
$$

in which $l_{i}$ means the actuator' length. $\boldsymbol{a}_{i}^{p}$ is the upper joint point's radius vector in body framework. $\boldsymbol{b}_{i}$ is the lower joint point's radius vector in inertia framework.

The upper platform's position can be achieved from the analysis of the forward kinematic using the given leg's length. In this section, the upper platform's position is obtained with the Newton-Raphson iterative algorithm. This algorithm's iterative sequence is described as

$$
\bar{q}_{j+1}=\bar{q}_{j}+\boldsymbol{J}_{\bar{q}}{ }^{-1}\left(l_{m}-l_{j}\right)
$$

where $l_{m}$ means the measured limb position.

2.3. Actuator Dynamics. The parallel robotic manipulator is driven by 12 miniature electrical actuators. In this paper, the friction of the actuator is ignored. Thus, the force equilibrium equation can be described as

$$
\boldsymbol{F}_{p}=\boldsymbol{F}_{a}
$$

In which $\boldsymbol{F}_{p}$ means the actuator's output force. $\boldsymbol{F}_{a}$ is measured by the force sensor.
Based on the motor output force principle and parameters of the motor, the actuator's output force $\boldsymbol{F}_{p}$ is expressed as

$$
\boldsymbol{F}_{p}=k_{t} \boldsymbol{I}_{t}
$$

in which $k_{t}$ means the motor's force coefficient. $\boldsymbol{I}_{t}$ denotes the equivalent current resulted from the current loop and this equivalent current can be expressed as

$$
I_{t}=\frac{1}{\tau_{0} s+1} \boldsymbol{U}
$$

in which $\tau_{0}$ means the time constant of the current loop and $\boldsymbol{U}$ denotes the input of the current loop that is described as

$$
U_{i}=U_{r i}-k_{e} i_{i}
$$

in which $k_{e}$ shows the equivalent electromotive force (EMF) coefficient. $U_{r i}$ means the $i$ th motor's control voltage.

\section{Control Design}

3.1. Active Force Control Strategy in Traditional Physical Space. The traditional physical control space consists of workspace and joint space. Since it is difficult to measure poses of endeffector, joint space control strategy (JSCS) is widely used in engineering. Therefore, this paper just presents the JSCS control method in traditional physical space in Figure 2.

In Figure 2, $\boldsymbol{\tau}_{d}$ is the desired force of the system in task space and $\boldsymbol{V}$ is the control law in traditional joint space.

Because the typical PID control approach is applied extensively in engineering, a classical P control strategy is used as the force controller.

$$
\boldsymbol{V}=k_{p} \boldsymbol{e}=k_{p}\left(\boldsymbol{F}_{a d}-\boldsymbol{F}_{a}\right)
$$

in which $k_{p}$ means the $\mathrm{P}$ control gain and is tuned in experimental process. $\boldsymbol{F}_{a d}$ is the desired force of the actuator.

3.2. Active Force Control Strategy in Modal Space. Because of the strong dynamic coupling of parallel robotic manipulator in joint space and task space, it is impossible to control multiple input and multiple output (MIMO) dynamic system independently. In order to decouple the dynamic coupling system and improve the system performance, modal space decoupling technique is used in this paper. Each channel control parameters are tuned in experimental process independently in modal space [36]. As shown in Figure 3, the 


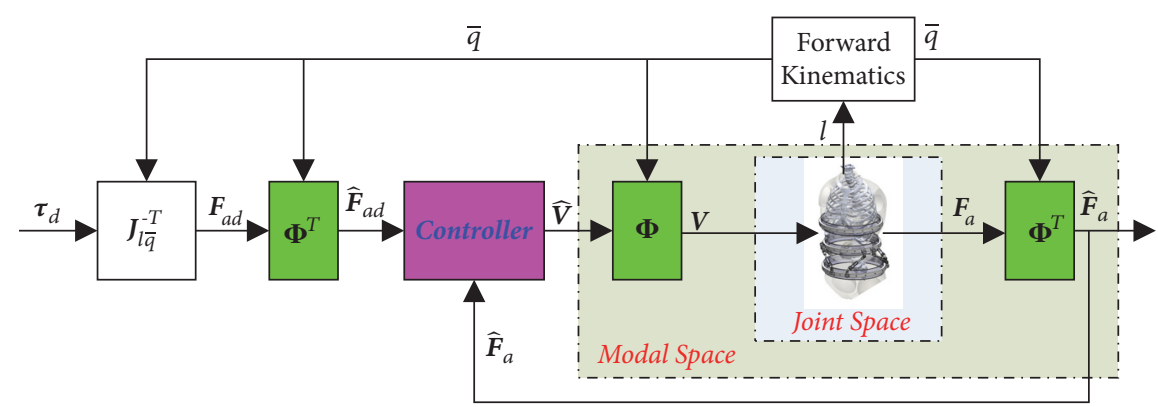

FIGURE 3: Active force control strategy in modal space.

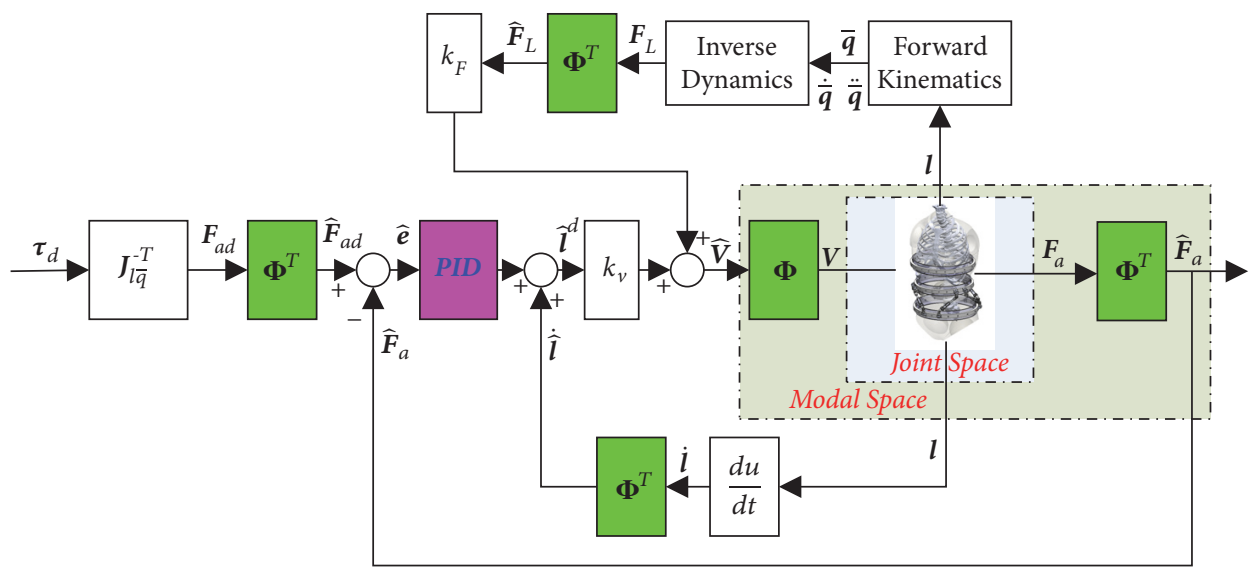

FIGURE 4: Modal space dynamic feed-forward (MSDF) force control strategy.

MIMO system is controlled in modal space where each channel is controlled independently.

In Figure 3, $\Phi$ means the modal translation matrix. $\Phi^{T}$ is the transpose of matrix $\boldsymbol{\Phi} . \widehat{\boldsymbol{F}}_{a d}$ denotes the reference modal driven force. $\widehat{\boldsymbol{F}}_{a}$ means the measured feedback force from the load cell. $\widehat{V}$ denotes the modal input voltage. $\boldsymbol{V}$ is the parallel mechanism's control input and $\boldsymbol{F}_{a}$ is the feedback force of the intelligent manipulator. The output of the modal controller is $\widehat{\boldsymbol{V}}$ and $\widehat{\boldsymbol{F}}_{a}$ is the modal feedback force.

Each force channel is controlled in modal space independently [36]. For revealing the property, the modal control law adopts the typical $\mathrm{P}$ control method

$$
\widehat{\boldsymbol{V}}=\widehat{\boldsymbol{K}}_{p} \widehat{\boldsymbol{e}}=\left[\begin{array}{cccc}
\widehat{k}_{1} & & & \\
& \widehat{k}_{2} & & \\
& & \ddots & \\
& & & \widehat{k}_{6}
\end{array}\right]\left[\begin{array}{c}
\widehat{F}_{a d 1}-\widehat{F}_{a 1} \\
\widehat{F}_{a d 2}-\widehat{F}_{a 2} \\
\vdots \\
\widehat{F}_{a d 6}-\widehat{F}_{a 6}
\end{array}\right]
$$

in which $\widehat{k}_{i}$ denotes the ith $\mathrm{P}$ control gain. $\widehat{\boldsymbol{e}}$ means the modal control error, $\widehat{\boldsymbol{e}}=\widehat{\boldsymbol{F}}_{a d}-\widehat{\boldsymbol{F}}_{a}$

\subsection{Dynamics Feed-Forward Active Force Control Strategy in} Modal Space. For improving performances of the parallel mechanism, the MSDF active force control strategy is presented in this paper. MSDF control strategy is constructed by adding a dynamic feed-forward to the modal space PID (MSPID) control method. MSDF control strategy can greatly improve the dynamic trajectory tracking performance of the parallel robotic manipulator without affecting the robust stability of MSPID control. The principle of MSDF is shown in Figure 4.

In Figure $4, k_{v}$ means the feed-forward gain of velocity. $k_{F}$ denotes the dynamic feed-forward gain. $\boldsymbol{F}_{L}$ is the compensation force of the system. $\widehat{\boldsymbol{F}}_{L}$ is the modal compensation force. $\widehat{l}^{d}$ is the velocity error of actuator in modal space. $\dot{\vec{l}}$ is the modal velocity of actuator.

According to the feed-forward strategy, this paper proposed the modal control law without destroying MSPID system stability.

$$
\begin{aligned}
\widehat{\boldsymbol{V}} & =k_{v} \widehat{\boldsymbol{l}}^{d}+k_{F} \widehat{\boldsymbol{F}}_{L} \\
\widehat{\boldsymbol{l}}^{d} & =\dot{\hat{\boldsymbol{l}}}+\widehat{\boldsymbol{K}}_{p} \widehat{\boldsymbol{e}}=\dot{\hat{\boldsymbol{l}}}+\widehat{\boldsymbol{K}}_{p}\left(\widehat{\boldsymbol{F}}_{a d}-\widehat{\boldsymbol{F}}_{a}\right) \\
\widehat{\boldsymbol{F}}_{L} & =\boldsymbol{\Phi}^{T}\left(\boldsymbol{G}_{l}+\boldsymbol{M}_{l} \ddot{\boldsymbol{l}}\right)
\end{aligned}
$$

Equation (26) is expressed as

$$
\widehat{\boldsymbol{F}}_{L}=\widehat{\boldsymbol{G}}_{l}+\widehat{\boldsymbol{M}}_{l} \ddot{\overrightarrow{\boldsymbol{l}}}
$$

in which $\widehat{\boldsymbol{M}}_{l}$ is the mass matrix modal space, $\widehat{\boldsymbol{M}}_{l}=$ $\operatorname{diag}\left\{\widehat{M}_{l 1} \quad \ldots \quad \widehat{M}_{l 6}\right\} . \widehat{\mathbf{G}}_{l}$ means the gravity term in modal space, $\widehat{G}_{l}=\left[\begin{array}{lll}\widehat{G}_{l 1} & \cdots & \widehat{G}_{l 6}\end{array}\right]$. 
According to the Laplace transformation, (24), (25), and (27) are described as

$$
\begin{aligned}
\widehat{\boldsymbol{V}} & =k_{v} \widehat{\boldsymbol{l}}^{d}(s)+k_{F} \widehat{\boldsymbol{F}}_{L}(s) \\
\widehat{\boldsymbol{l}}^{d} & =s \widehat{\boldsymbol{l}}+\widehat{\boldsymbol{K}}_{p}\left(\widehat{\boldsymbol{F}}_{a d}-\widehat{\boldsymbol{F}}_{a}\right) \\
\widehat{\boldsymbol{F}}_{L} & =\widehat{\boldsymbol{G}}_{l}+s^{2} \widehat{\boldsymbol{M}}_{\boldsymbol{l}} \widehat{\boldsymbol{l}}
\end{aligned}
$$

Combing (29) and (30) with (28), the modal force control law is achieved

$$
\begin{aligned}
\widehat{\boldsymbol{V}}(s)= & k_{v} \widehat{\boldsymbol{K}}_{p}\left[\left(k_{F} \widehat{\boldsymbol{K}}_{p}^{-1} \widehat{\boldsymbol{M}}_{l} s^{2}+\widehat{\boldsymbol{K}}_{p}^{-1} s\right) \widehat{\boldsymbol{l}}+\left(\widehat{\boldsymbol{F}}_{a d}-\widehat{\boldsymbol{F}}_{a}\right)\right] \\
& +k_{F} \widehat{\boldsymbol{G}}_{l}
\end{aligned}
$$

Equation (31) is expressed as

$$
\begin{aligned}
\widehat{V}_{i}(s)= & k_{v} \widehat{k}_{p i}\left[\left(\frac{k_{F} \widehat{M}_{l i}}{\widehat{k}_{p i}} s+\frac{1}{\widehat{k}_{p i}}\right) \dot{\hat{l}}_{i}+\left(\widehat{F}_{a d i}-\widehat{F}_{a i}\right)\right] \\
& +k_{F} \widehat{G}_{l i}
\end{aligned}
$$

As shown in (32), according to the MSPID control method, the MSDF control law of each channel consists of a gravity compensation term and a velocity feedback differentiation loop independently. The controller design and parameters adjustment of MSDF are carried out in each channel independently. According to the performance of each modal channel, the designed controller makes the performance of each channel optimal in modal space, thus achieving the best overall control performance of the parallel robotic manipulator system.

\section{Stability Analysis}

Stability shows the critical property of the parallel mechanism. Since the dynamic feed-forward control strategy does not destroy the whole system stability, this paper just derives the MSPID system stability.

According to (19), (20), and (21), it can be obtained

$$
k_{t}^{\prime}\left(\boldsymbol{U}_{r}-k_{e} l\right)=\boldsymbol{F}_{p}
$$

where $k_{t}^{\prime}=k_{t}\left(1 /\left(\tau_{0} s+1\right)\right)$

Based on Laplace transformation, (34) is described as

$$
s l=\frac{1}{k_{e}}\left(\boldsymbol{U}_{r}-\frac{1}{k_{t}^{\prime}} \boldsymbol{F}_{p}\right)
$$

Based on (14) and (18), EQ (35) is obtained

$$
\boldsymbol{M}_{l}(l) \ddot{l}+\boldsymbol{C}_{l}(l, \dot{l}) \dot{l}+\boldsymbol{G}_{l}(l)+\boldsymbol{F}_{c l}=\boldsymbol{F}_{p}
$$

Since the parallel robotic spine brace works in low speed area, the centrifugal term $C_{l}$ is so small that can be ignored [36]. Thus, (35) is expressed as

$$
\boldsymbol{M}_{l}(l) \ddot{l}+\boldsymbol{G}_{l}(l)+\boldsymbol{F}_{c l}=\boldsymbol{F}_{p}
$$

Based on Laplace transformation, (37) is obtained

$$
s^{2} \boldsymbol{M}_{l}(l) l=\boldsymbol{F}_{p}-\left(\boldsymbol{G}_{l}(l)+\boldsymbol{F}_{c l}\right)
$$

Combine (34) with (37)

$$
s \boldsymbol{M}_{l}(l) \boldsymbol{U}_{r}=\left(k_{e} \boldsymbol{I}+\frac{1}{k_{t}^{\prime}} s \boldsymbol{M}_{l}(l)\right) \boldsymbol{F}_{p}-k_{e}\left(\boldsymbol{G}_{l}(l)+\boldsymbol{F}_{c l}\right)
$$

Based on the transformational relation from the joint space to the modal space, it can be obtained

$$
\boldsymbol{M}_{l}=\boldsymbol{\Phi} \widehat{\boldsymbol{M}}_{l} \boldsymbol{\Phi}^{T}
$$

Combine (39) with (38)

$$
\begin{aligned}
\boldsymbol{\Phi}\left(s \widehat{\boldsymbol{M}}_{l}\right) \boldsymbol{\Phi}^{T} \boldsymbol{U}_{r}= & \boldsymbol{\Phi}\left(k_{e} \boldsymbol{I}+\frac{1}{k_{t}^{\prime}} s \widehat{\boldsymbol{M}}_{l}\right) \boldsymbol{\Phi}^{T} \boldsymbol{F}_{p} \\
& -k_{e}\left(\boldsymbol{G}_{l}(l)+\boldsymbol{F}_{c l}\right)
\end{aligned}
$$

where

$$
\begin{aligned}
& \widehat{\boldsymbol{U}}_{r}=\boldsymbol{\Phi}^{T} \boldsymbol{U}_{r} \\
& \widehat{\boldsymbol{F}}_{p}=\boldsymbol{\Phi}^{T} \boldsymbol{F}_{p}
\end{aligned}
$$

in which $\widehat{\boldsymbol{U}}_{r}$ is the modal motor voltage. $\widehat{\boldsymbol{F}}_{p}$ is the output force of actuator in modal space.

Based on (40) and (41), (42) is described as

$$
\left(s \widehat{\boldsymbol{M}}_{l}\right) \widehat{\boldsymbol{U}}_{r}=\left(k_{e} \boldsymbol{I}+\frac{1}{k_{t}^{\prime}} s \widehat{\boldsymbol{M}}_{l}\right) \widehat{\boldsymbol{F}}_{p}-k_{e} \boldsymbol{\Phi}^{T}\left(\boldsymbol{G}_{l}(l)+\boldsymbol{F}_{c l}\right)
$$

According to (18) and (42), the transfer function from $\widehat{\boldsymbol{U}}_{r}$ to $\widehat{\boldsymbol{F}}_{a}$ can be obtained

$$
\frac{k_{t} \widehat{M}_{l i} s}{\tau_{0} \widehat{M}_{l i} s^{2}+\widehat{M}_{l i} s+k_{t} k_{e}} \widehat{U}_{r i}=\widehat{F}_{a i}
$$

Because of $\widehat{U}_{r i}=\widehat{V}_{i}(43)$ is expressed as

$$
\frac{k_{t} \widehat{M}_{l i} s}{\tau_{0} \widehat{M}_{l i} s^{2}+\widehat{M}_{l i} s+k_{t} k_{e}} \widehat{V}_{i}=\widehat{F}_{a i}
$$

Based on (23) and (44), the open-loop transfer function of the MSPID system can be obtained

$$
\begin{aligned}
\widehat{G}_{i}(s) & =\frac{\widehat{F}_{a i}}{\widehat{F}_{a d i}} \\
& =\frac{\left(k_{p i} \widehat{M}_{l i} / k_{e}\right) s}{s^{2} /\left(k_{t} k_{e} / \tau_{0} \widehat{M}_{l i}\right)+2 \widehat{\xi}_{i} s / \sqrt{k_{t} k_{e} / \tau_{0} \widehat{M}_{l i}}+1}
\end{aligned}
$$

in which $\hat{\xi}_{i}$ means the damping coefficient, $\widehat{\xi}_{i}=$ $1 / 2 \sqrt{k_{t} k_{e} \tau_{0} / \widehat{M}_{l i}}$. Equation (45) is described as

$$
\widehat{G}_{i}(s)=\frac{\left(k_{p i} \widehat{M}_{l i} / k_{e}\right) s}{s^{2} / \widehat{\omega}_{n i}^{2}+2 \xi_{i} s / \widehat{\omega}_{n i}+1}
$$




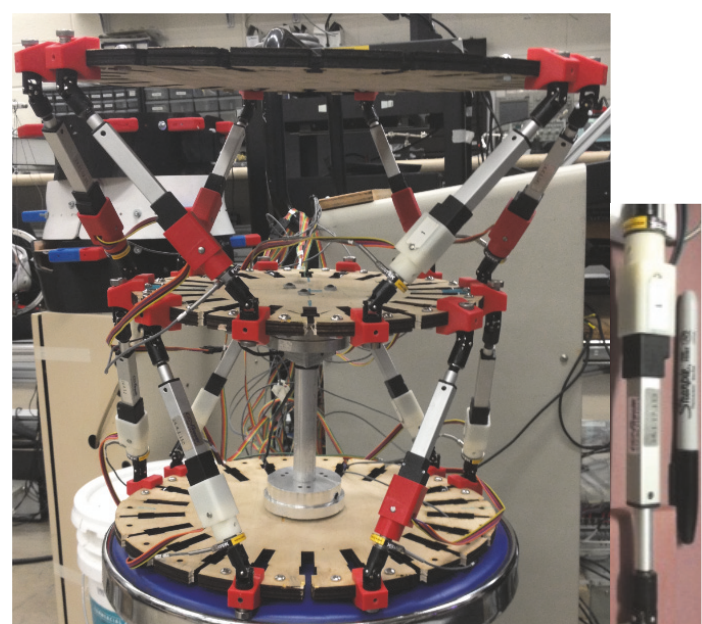

FIGURE 5: Experimental parallel spine brace.

in which $\widehat{\omega}_{n i}$ denotes the system's natural frequency, $\widehat{\omega}_{n i}=$ $\sqrt{k_{t} k_{e} / \tau_{0} \widehat{M}_{l i}}$

Based on the Bode stability criterion, for guaranteeing the MSPID system stability, the system control parameters should satisfy (47).

$$
\frac{k_{p i} \widehat{M}_{l i}}{k_{e}}<2 \widehat{\xi}_{i} \widehat{\omega}_{n i}
$$

As shown in (47), in order to guarantee the MSPID system stability, the modal gain matrix $\widehat{\boldsymbol{K}}_{p}$ should be chosen appropriately. Thus, the MSDF system is robustly stable.

\section{Experiment}

Using the proposed MSDF force control method, some experiments are carried out for evaluating performances of the intelligent robotic system. Compared with the MSPID force control algorithm, the MSDF control strategy can achieve better performances.

5.1. Experiment Setup. For implementing and evaluating performances of the presented MSDF force control method, this section builds the parallel intelligent mechanism in Figure 5. This parallel system has several features: (1) 12 linear electric cylinders, (2) a real-time controller, and (3) a monitor computer. The force given signals are provided by the monitor computer. Besides, the force controller is running in a realtime controller. Geometric parameters of the brace system are shown in Table 1 and the sampling time for the experimental system is set to $20 \mathrm{~ms}$.

5.2. Experiment Results. Scoliosis is a 3D deformity. In order to achieve better orthopedic effect, this paper proposed an MSDF control structure for the robotic manipulator. For verifying the performance of the system with this presented
TABLE 1: Parameters of the experimental parallel intelligent manipulator.

\begin{tabular}{lc}
\hline Parameters & Values \\
\hline Ellipse's long distance of upper joint $(\mathrm{mm})$ & 150 \\
Ellipse's short distance of upper joint $(\mathrm{mm})$ & 142 \\
Ellipse's long distance of lower joint $(\mathrm{mm})$ & 170 \\
Ellipse's long distance of lower joint $(\mathrm{mm})$ & 149 \\
Maximal stroke of linear electric cylinder $(\mathrm{mm})$ & 50 \\
Initial length of linear electric cylinder $(\mathrm{mm})$ & 160 \\
Masses of moving platform and joints $(\mathrm{Kg})$ & 0.33 \\
\hline
\end{tabular}

control method, several force signals are given to the robotic system. The modal space $\mathrm{P}$ controllers are tuned to be

$$
\widehat{\boldsymbol{K}}_{p}=\operatorname{diag}\left[\begin{array}{llllll}
16 & 16 & 16 & 16 & 16 & 16
\end{array}\right]
$$

The dynamic feed-forward control parameters $k_{v}$ and $k_{F}$ are tuned to be

$$
\begin{aligned}
& k_{v}=\operatorname{diag}\left[\begin{array}{llllll}
5.5 & 5.5 & 5.5 & 5.5 & 5.5 & 5.5
\end{array}\right] \\
& k_{F}=\operatorname{diag}\left[\begin{array}{llllll}
1.6 & 1.6 & 1.6 & 1.6 & 1.6 & 1.6
\end{array}\right]
\end{aligned}
$$

As shown in Figure 6, step signals (surge: 8 N, sway: $8 \mathrm{~N}$, heave: $10 \mathrm{~N}$, roll: $1.5 \mathrm{Nm}$, pitch: $1.5 \mathrm{Nm}$, yaw: 1.5 $\mathrm{Nm}$ ) are given to the robotic system in all six directions to detect the performance of the system. It can be seen from Figure 6 that the celerity of MSDF controller is superior to MSPID controller. In addition, the steady-state error of MSDF controller is smaller than that of MSPID controller. The maximal steady-state error is $0.1 \mathrm{~N}$ in linear directions and $0.05 \mathrm{Nm}$ in angular directions with the proposed MSDF control strategy, $0.5 \mathrm{~N}$ in linear directions and $0.1 \mathrm{Nm}$ in angular directions under the traditional MSPID control method. Furthermore, the dynamic system with the MSDF controller responds to desired force signals more quickly than that of the MSPID controller in all six directions.

As shown in (50a), (50b), (50c), (50d), (50e), and (50f) the reference special force signals are exerted on the robotic spine brace system respectively. Responses to the desired special force signals are shown in Figures 7 and 8 depicting the dynamic errors of the robotic system.

$$
\begin{aligned}
F_{x}= & 3 \sin (0.377 t)+4 \sin (0.314 t)+5 \sin (0.253 t) \\
F_{y}= & 4 \sin (0.314 t)+3 \sin (0.188 t)+5 \sin (0.253 t) \\
F_{z}= & 2 \sin (0.314 t)+3 \sin (0.188 t)+5 \sin (0.253 t) \\
M_{x}= & 0.8 \sin (0.440 t)+1.0 \sin (0.377 t) \\
& +1.2 \sin (0.314 t) \\
M_{y}= & 0.8 \sin (0.377 t)+1.0 \sin (0.314 t) \\
& +1.2 \sin (0.440 t) \\
M_{z}= & 0.8 \sin (0.377 t)+1.0 \sin (0.314 t) \\
& +1.2 \sin (0.440 t)
\end{aligned}
$$



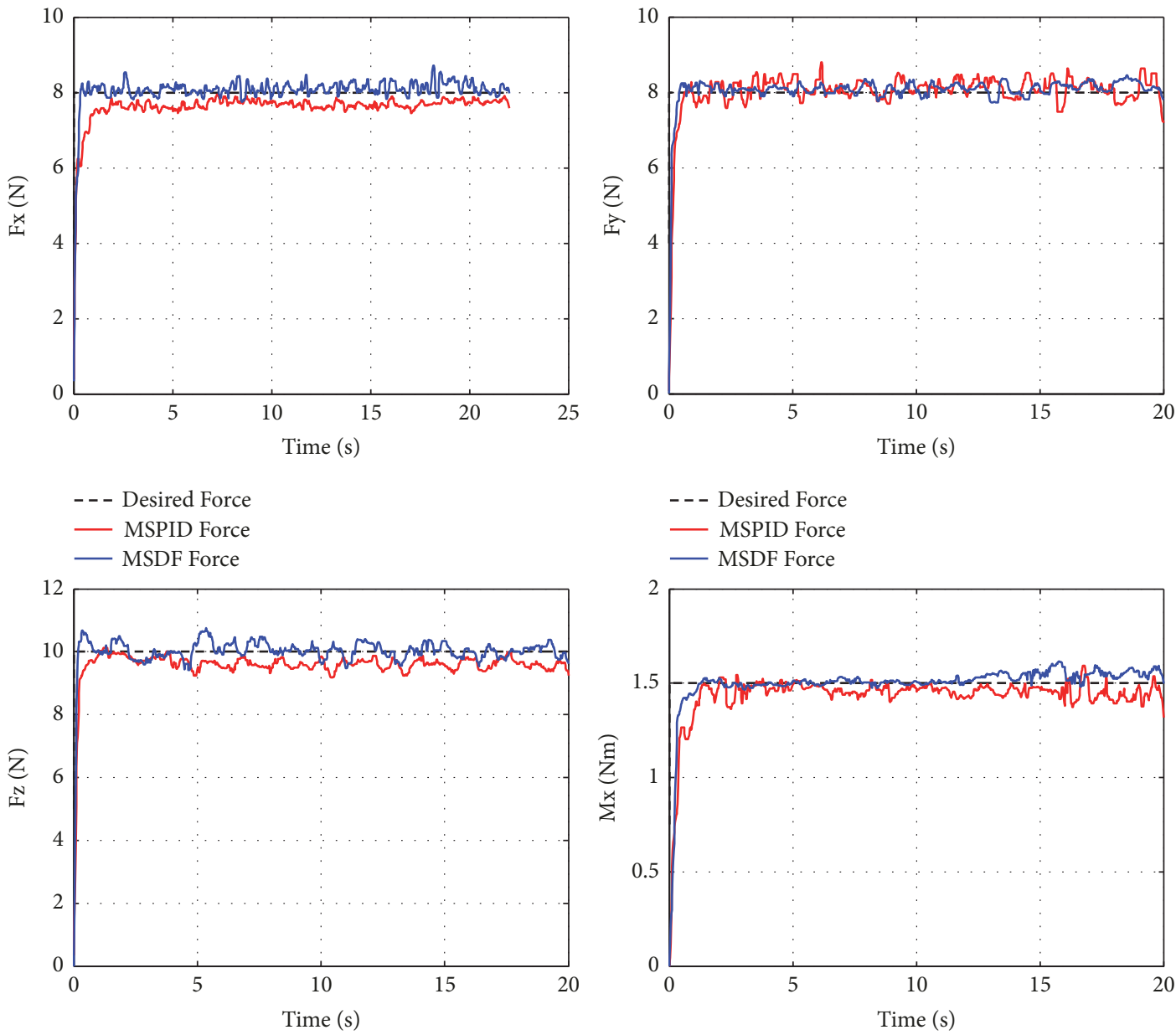

- - - Desired Force

- MSPID Force

— MSDF Force

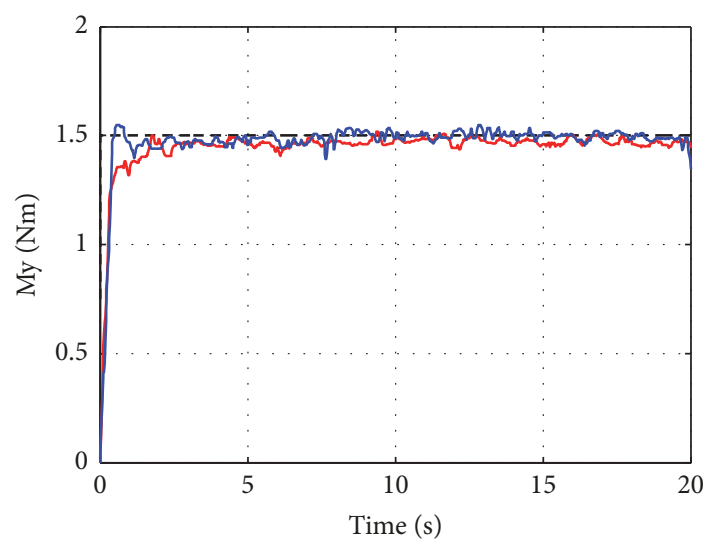

$$
\begin{aligned}
& - \text { - Desired Force } \\
& \text { — MSPID Force } \\
& \text { — MSDF Force }
\end{aligned}
$$
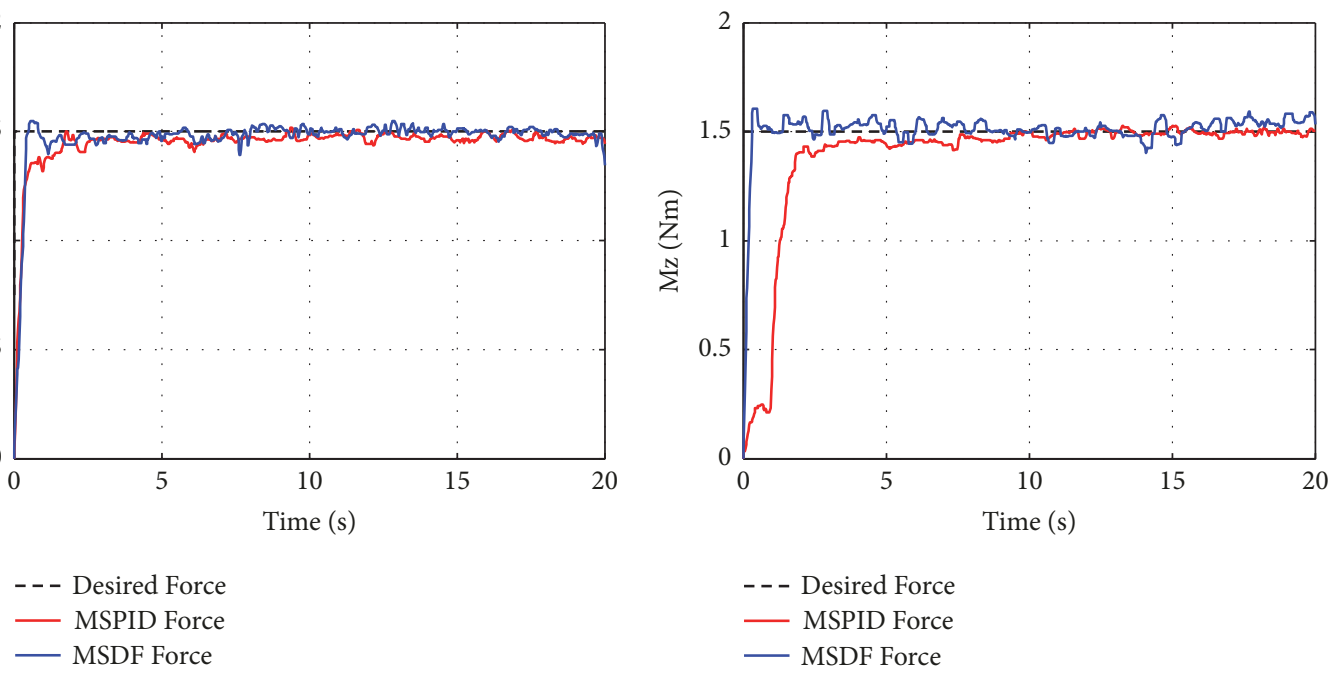

FIGURE 6: Responses to the desired step force signals with MSPID and MSDF controllers. 

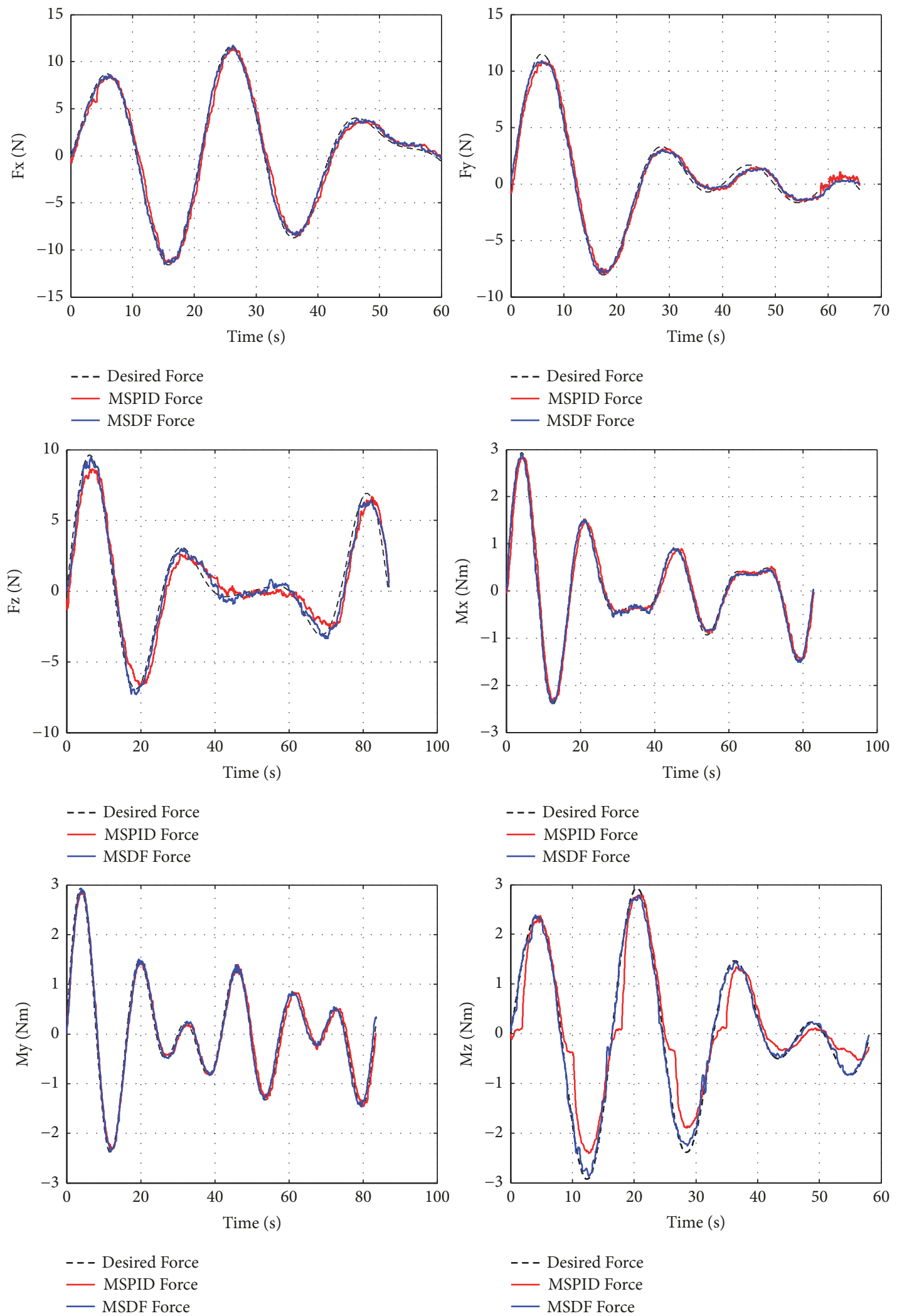

FIgURE 7: Responses to the desired special force signals with MSPID and MSDF controllers. 

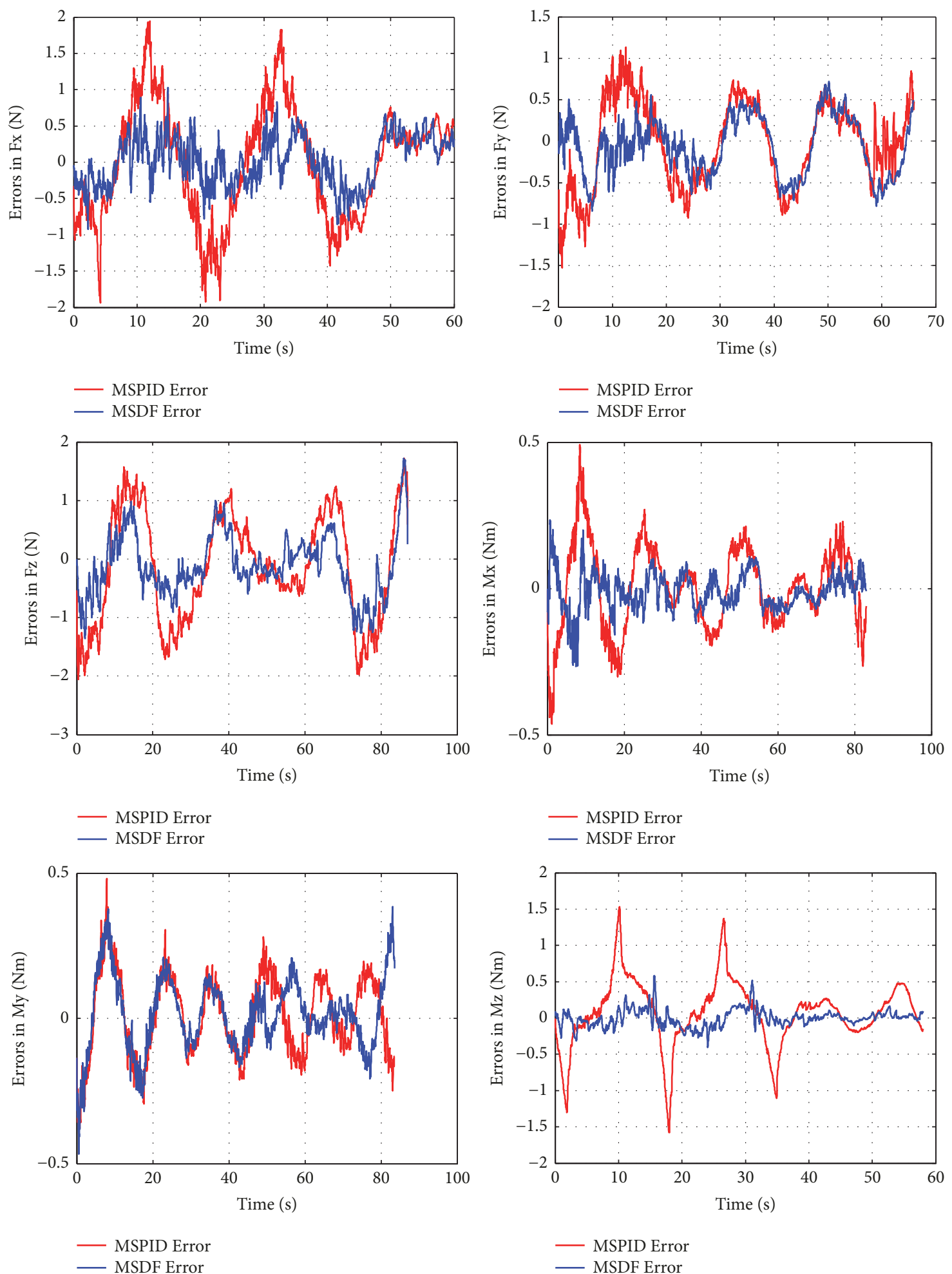

FIGURE 8: Dynamic tracking errors of parallel robotic manipulator with MSPID and MSDF controllers.

As shown in Figures 7 and 8, the dynamic response performance of the proposed MSDF controller in time domain is much better than that of MSPID controller in all directions. In comparison to the typical MSPID control method, the attenuation amplitude reduces $21 \%$ at least and the lag phase is $47 \%$ at least with the presented MSDF force control strategy. Thus, the designed MSDF force control strategy can improve the control performance and dynamic tracking accuracy of the parallel robotic manipulator. 

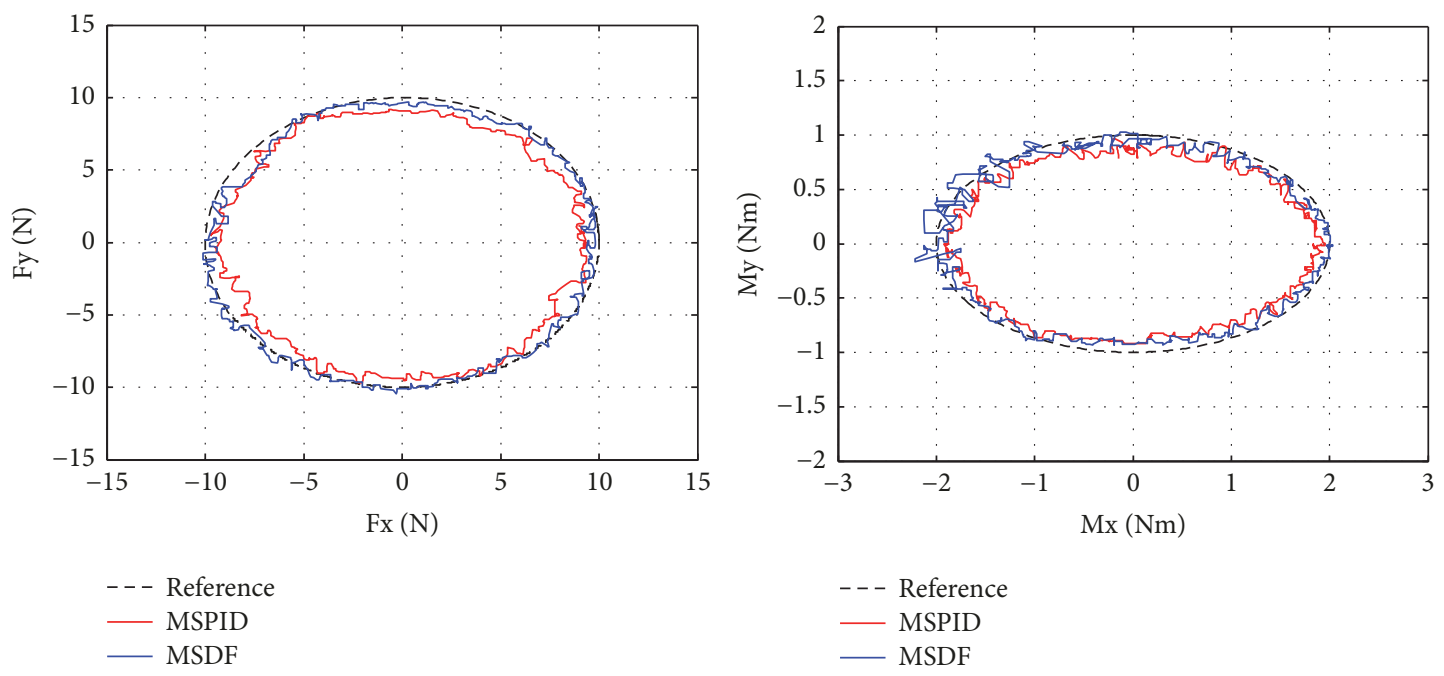

Figure 9: Responses to the combined force commands with MSPID and MSDF controllers.

However, the above experiments are all single-DOF. Thus, the combine force signals are desired for evaluating the performance of the robotic brace system. The desired force signals are applied on the parallel manipulator in EQ (51a), (51b), (51d), and (51e) and the responses are shown in Figure 9.

$$
\begin{aligned}
F_{x} & =10 \cos (0.377 t) \\
F_{y} & =10 \sin (0.377 t) \\
M_{x} & =2.0 \sin (0.314 t) \\
M_{y} & =1.0 \cos (0.314 t)
\end{aligned}
$$

As can be seen from Figure 9, the robotic brace responds to the desired combined force signals much better under the MSDF control strategy than that of MSPID control strategy. MSDF controller has smaller track errors than the MSPID controller.

As can be deduced from Figures 6-9, the single freedom dynamic tracking performance of the robotic brace system with the presented MSDF controller is superior to that with the MSPID controller and the composite dynamic trajectory tracking performance is also better than the MSPID control strategy. MSDF controller not only solves the dynamic coupling in traditional psychical space but compensates the gravity of the system. Besides, the steady-state error of the system with MSDF control method is also smaller than that of the MSPID control strategy. Thus, MSDF control can track the desired dynamic trajectory more quickly and accurately and applying high-precision 3D corrective forces on human spine.

\section{Conclusion}

This paper investigates modal space dynamic feed-forward control structure for parallel robotic manipulator, to overcome those boring problems. The system models are developed, including the dynamic model of the robotic spine brace, the actuator's dynamic model, and the forward kinematics and inverse kinematics models of the system. Besides, the stability of the MSDF control strategy is analyzed and the novel controller reveals excellent robustness. Based on the typical PID force control method, those boring problems of parallel robotic manipulator greatly affect performances of the intelligent system in traditional physical space. For those boring problems, the MSDF active force control strategy is designed based on the dynamic feed-forward control algorithm and the modal decoupling approach. In order to verify performances of the parallel mechanism, experimental given signals are applied on the intelligent system with the proposed MSDF force control method. According to those experimental results, the MSDF force control algorithm can track the desired force dynamic trajectory more quickly and accurately, and apply high-precision 3D corrective forces on human spine. Compared with MSPID control method, the proposed MSDF control strategy presents excellent performance for the robotic spine brace. The system is stable and the state-errors of the system because of the boring problems tend to zero asymptotically.

Although performance of the system is excellent using the novel MSDF control strategy, there are some improvements needed to be done for this robotic spine brace in the future. Currently, this control strategy ignores disturbers from system nonlinearity, modeling errors, and other external interferences. In the future, an antidisturbance observer strategy should be developed to improve the performance and robustness of this robotic brace system. Furthermore, the current control method does not consider the model of human spine which is important for the control system. Thus, one of the next steps is building the three-dimensional model of human spine.

\section{Data Availability}

The Matlab Source Code data used to support the findings of this study are available from the corresponding author upon request. 


\section{Conflicts of Interest}

The authors declare that there are no conflicts of interest regarding the publication of this paper.

\section{Authors' Contributions}

Mr. Niu provided the ideas for this article and wrote this paper. Prof. Yang helped build the test platform and provided financial support for this paper. Dr. Tian made contributions to data collection and English check of the paper. Dr. Li made contributions to formula deduction of the paper and the revision of the review comments. Prof. Han made contributions to English check and collection of references and typesetting of this paper.

\section{Acknowledgments}

The authors would also like to thank Dr. Sunil K Agrawal for the help of putting forward the concept of this robotic structure. The work was supported by the National Natural Science Foundation of China (grant number 51305095) and the Natural Science Foundation of Heilongjiang Province (grant number QC2013C045).

\section{References}

[1] S. L. Weinstein, L. A. Dolan, J. C. Cheng, A. Danielsson, and J. A. Morcuende, "Adolescent idiopathic scoliosis," The Lancet, vol. 371, no. 9623, pp. 1527-1537, 2008.

[2] R. S. Fayssoux, R. H. Cho, and M. J. Herman, "A history of bracing for idiopathic scoliosis in north America," Clinical Orthopaedics and Related Research, vol. 468, no. 3, pp. 654-664, 2010.

[3] M. A. Asher and D. C. Burton, "Adolescent idiopathic scoliosis: natural history and long term treatment effects," Scoliosis, vol. 1, pp. 2-2, 2006.

[4] S. Negrini, S. Minozzi, J. Bettany-Saltikov, F. Zaina, N. Chockalingam, and T. B. Grivas, Braces for Idiopathic Scoliosis in Adolescents, John Wiley and Sons Ltd, 2007.

[5] H.-R. Weiss and M. Werkmann, "Retraction: Soft braces in the treatment of Adolescent Idiopathic Scoliosis (AIS) - Review of the literature and description of a new approach," Scoliosis, vol. 8, no. 1, p. 7, 2013.

[6] J.-H. Park, P. Stegall, and S. K. Agrawal, "Dynamic brace for correction of abnormal postures of the human spine," in Proceedings of the 2015 IEEE International Conference on Robotics and Automation, ICRA 2015, pp. 5922-5927, USA, May 2015.

[7] J. P. Merlet, "Parallel robots," Solid Mechanics and Its Applications, vol. 128, pp. 2091-2127, 2010.

[8] J. Wang and C. M. Gosselin, "Kinematic analysis and design of kinematically redundant parallel mechanisms," Journal of Mechanical Design, vol. 126, no. 1, pp. 109-118, 2004.

[9] Y. Lou, Y. Zhang, R. Huang, X. Chen, and Z. Li, "Optimization algorithms for kinematically optimal design of parallel manipulators," IEEE Transactions on Automation Science and Engineering, vol. 11, no. 2, pp. 574-584, 2014.

[10] T. Nozaki, T. Mizoguchi, and K. Ohnishi, "Decoupling strategy for position and force control based on modal space disturbance observer," IEEE Transactions on Industrial Electronics, vol. 61, no. 2, pp. 1022-1032, 2014.

[11] A. A. Allais, J. E. McInroy, and J. F. O’Brien, "Locally decoupled micromanipulation using an even number of parallel force actuators," IEEE Transactions on Robotics, vol. 28, no. 6, pp. 1323-1334, 2012.

[12] J. E. McInroy, J. F. OBrien, and A. A. Allais, "Designing micromanipulation systems for decoupled dynamics and control," IEEE/ASME Transactions on Mechatronics, vol. 20, no. 2, pp. 553-563, 2015.

[13] C. Gosselin and S. Foucault, "Dynamic point-to-point trajectory planning of a two-DOF cable-suspended parallel robot," IEEE Transactions on Robotics, vol. 30, no. 3, pp. 728-736, 2014.

[14] M. Carricato and J. Merlet, "Stability analysis of underconstrained cable-driven parallel robots," IEEE Transactions on Robotics, vol. 29, no. 1, pp. 288-296, 2013.

[15] J. Borras, F. Thomas, and C. Torras, "New geometric approaches to the analysis and design of stewart-gough platforms," IEEE/ASME Transactions on Mechatronics, vol. 19, no. 2, pp. 445-455, 2014.

[16] K. A. Arrouk, B. C. Bouzgarrou, and G. Gogu, "CAD-based unified graphical methodology for solving the main problems related to geometric and kinematic analysis of planar parallel robotic manipulators," Robotics and Computer-Integrated Manufacturing, vol. 37, pp. 302-321, 2016.

[17] J. Pile and N. Simaan, "Modeling, design, and evaluation of a parallel robot for cochlear implant surgery," IEEE/ASME Transactions on Mechatronics, vol. 19, no. 6, pp. 1746-1755, 2014.

[18] P. K. Jamwal, S. Q. Xie, S. Hussain, and J. G. Parsons, "An adaptive wearable parallel robot for the treatment of ankle injuries," IEEE/ASME Transactions on Mechatronics, vol. 19, no. 1, pp. 64-75, 2014.

[19] Y. Li and Q. Xu, "Dynamic modeling and robust control of a 3-PRC translational parallel kinematic machine," Robotics and Computer-Integrated Manufacturing, vol. 25, no. 3, pp. 630-640, 2009.

[20] A. M. Mohammed and S. Li, "Dynamic neural networks for kinematic redundancy resolution of parallel stewart platforms," IEEE Transactions on Cybernetics, vol. 46, no. 7, pp. 1538-1550, 2016.

[21] A. Dumlu and K. Erenturk, "Trajectory tracking control for a 3-DOF parallel manipulator using fractional-order $\operatorname{PI} \lambda \mathrm{D} \mu$ control," IEEE Transactions on Industrial Electronics, vol. 61, pp. 3417-3426, 2014.

[22] S.-H. Chen and L.-C. Fu, "Output feedback sliding mode control for a stewart platform with a nonlinear observer-based forward kinematics solution," IEEE Transactions on Control Systems Technology, vol. 21, no. 1, pp. 176-185, 2013.

[23] S. Mishra, P. S. Londhe, S. Mohan, S. K. Vishvakarma, and B. M. Patre, "Robust task-space motion control of a mobile manipulator using a nonlinear control with an uncertainty estimator," Computers and Electrical Engineering, vol. 67, pp. 729-740, 2018.

[24] S. A. Moosavian and E. Papadopoulos, "Modified transpose Jacobian control of robotic systems," Automatica, vol. 43, no. 7, pp. 1226-1233, 2007.

[25] S. Kim, Y. J. Shin, K. Kim, and S. Kim, "Design and control of robot manipulator with a distributed actuation mechanism," Mechatronics, vol. 24, no. 8, pp. 1223-1230, 2014.

[26] Y. Pi and X. Wang, "Observer-based cascade control of a 6DOF parallel hydraulic manipulator in joint space coordinate," Mechatronics, vol. 20, no. 6, pp. 648-655, 2010. 
[27] C. Yang, Q. Huang, H. Jiang, O. Ogbobe Peter, and J. Han, "PD control with gravity compensation for hydraulic 6-DOF parallel manipulator," Mechanism and Machine Theory, vol. 45, no. 4, pp. 666-677, 2010.

[28] S. Chen and L. Fu, "Observer-based backstepping control of a 6-dof parallel hydraulic manipulator," Control Engineering Practice, vol. 36, pp. 100-112, 2015.

[29] A. Noshadi, M. Mailah, and A. Zolfagharian, "Active force control of 3-RRR planar parallel manipulator," International Conference on Mechanical and Electrical Technology, pp. 77-81, 2010.

[30] T. K. Bera, R. Merzouki, B. Ould Bouamama, and A. K. Samantaray, "Force control in a parallel manipulator through virtual foundations," Proceedings of the Institution of Mechanical Engineers, Part I: Journal of Systems and Control Engineering, vol. 226, no. 8, pp. 1088-1106, 2012.

[31] A. Lopes and F. Almeida, "A force-impedance controlled industrial robot using an active robotic auxiliary device," Robotics and Computer Integrated Manufacturing, vol. 24, pp. 299-309, 2008.

[32] D. J. Goertzen and G. N. Kawchuk, "A novel application of velocity-based force control for use in robotic biomechanical testing," Journal of Biomechanics, vol. 42, no. 3, pp. 366-369, 2009.

[33] S. Wen, H. Yu, B. Zhang et al., "Fuzzy identification and delay compensation based on the force/position control scheme of the 5-DOF redundantly actuated parallel robot," International Journal of Fuzzy Systems, vol. 19, no. 1, pp. 124-140, 2017.

[34] C. Yang, Z. Qu, and J. Han, "Decoupled-space control and experimental evaluation of spatial electrohydraulic robotic manipulators using singular value decomposition algorithms," IEEE Transactions on Industrial Electronics, vol. 61, no. 7, pp. 3427-3438, 2014.

[35] C. Yang and J. Han, "Dynamic coupling analysis of a spatial 6-DOF electro-hydraulic parallel manipulator using a modal decoupling method," International Journal of Advanced Robotic Systems, vol. 10, p. 1, 2013.

[36] C. Yang, J. Zhao, L. Li, and S. K. Agrawal, "Design and implementation of a novel modal space active force control concept for spatial multi-DOF parallel robotic manipulators actuated by electrical actuators," ISA Transactions, vol. 72, pp. 273-286, 2018.

[37] M. W. Spong, Robot Dynamics and Control, John Wiley and Sons, 1989.

[38] L. Baron and J. Angeles, "The on-line direct kinematics of parallel manipulators under joint-sensor redundancy," IEEE Transactions on Robotics \& Automation, vol. 16, no. 1, pp. 12-19, 2000.

[39] S. Staicu, "Dynamics of the 6-6 Stewart parallel manipulator," Robotics and Computer-Integrated Manufacturing, vol. 27, no. 1, pp. 212-220, 2011. 


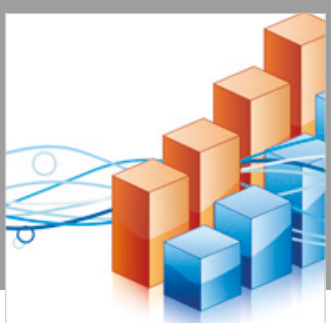

Advances in

Operations Research

\section{-n-m}
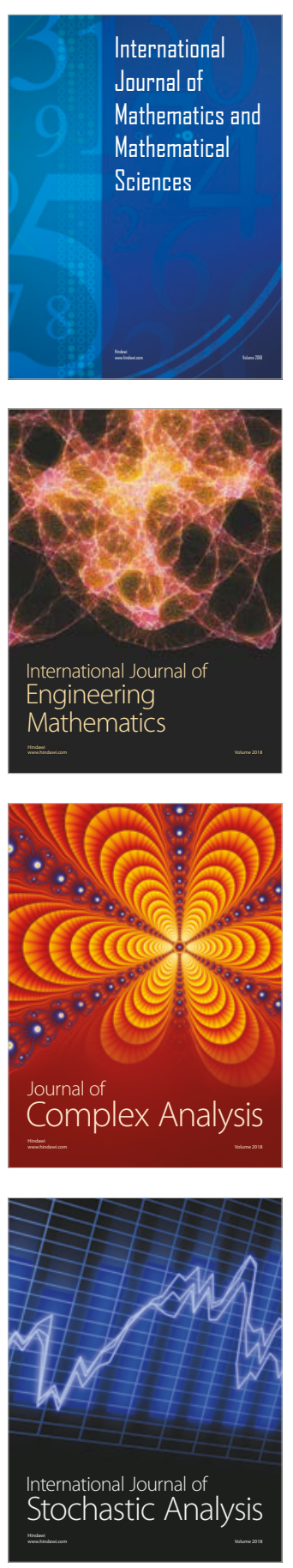
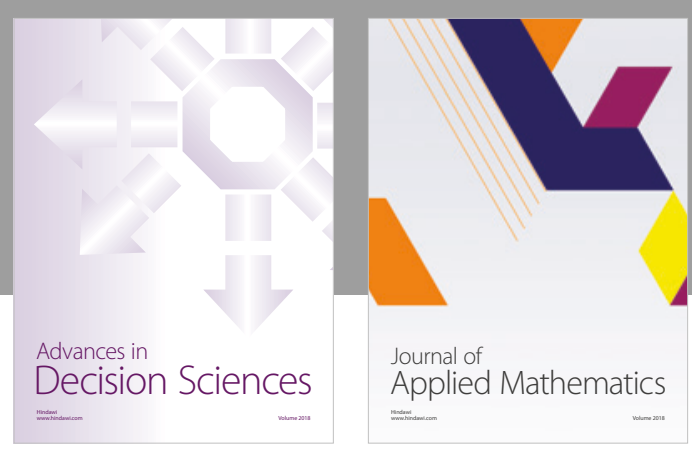

Journal of

Applied Mathematics
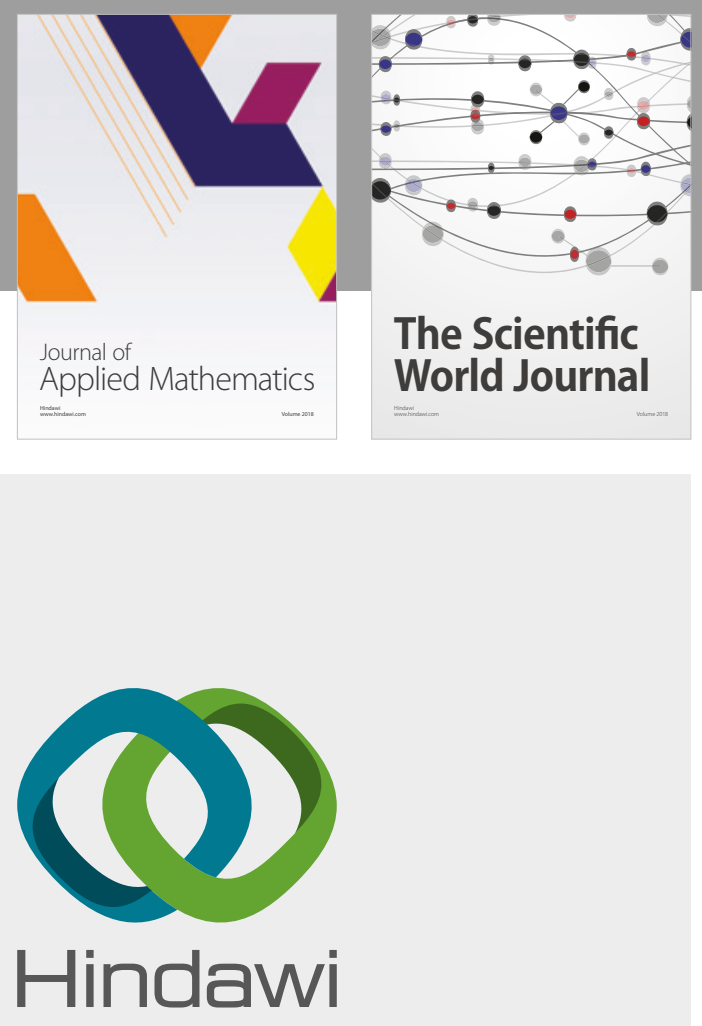

Submit your manuscripts at

www.hindawi.com

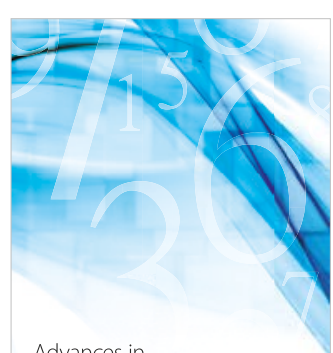

Advances in
Numerical Analysis
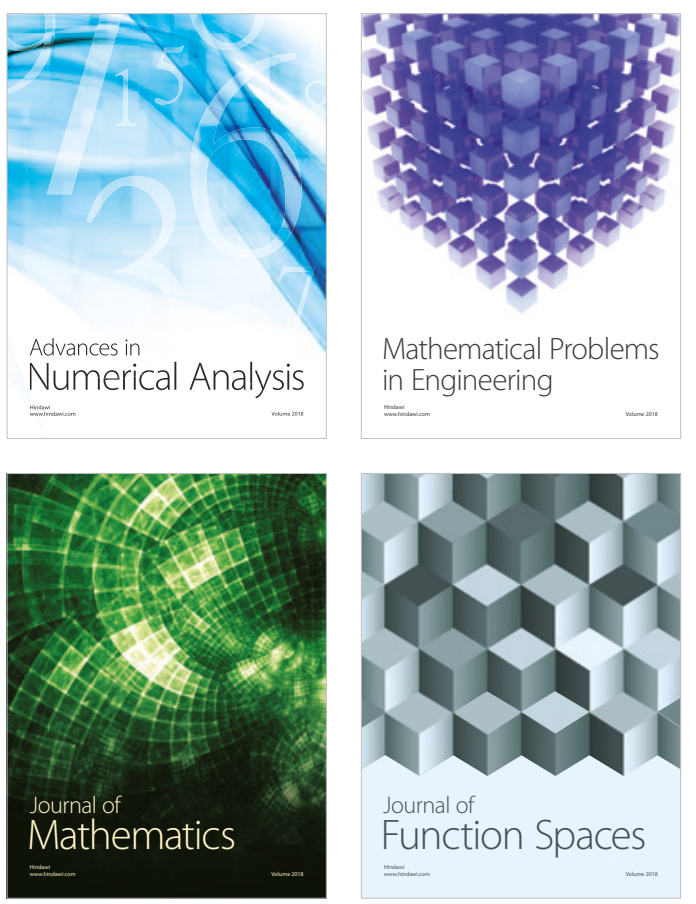

Mathematical Problems in Engineering

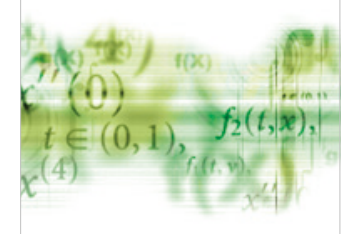

International Journal of

Differential Equations

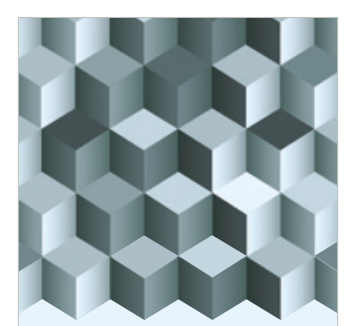

Journal of

Function Spaces

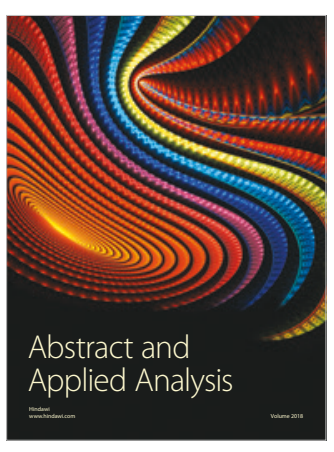

The Scientific

World Journal

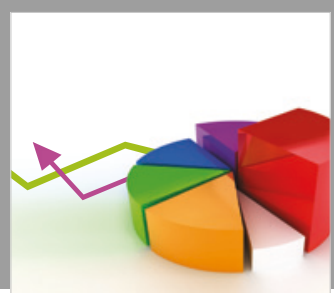

Journal of

Probability and Statistics
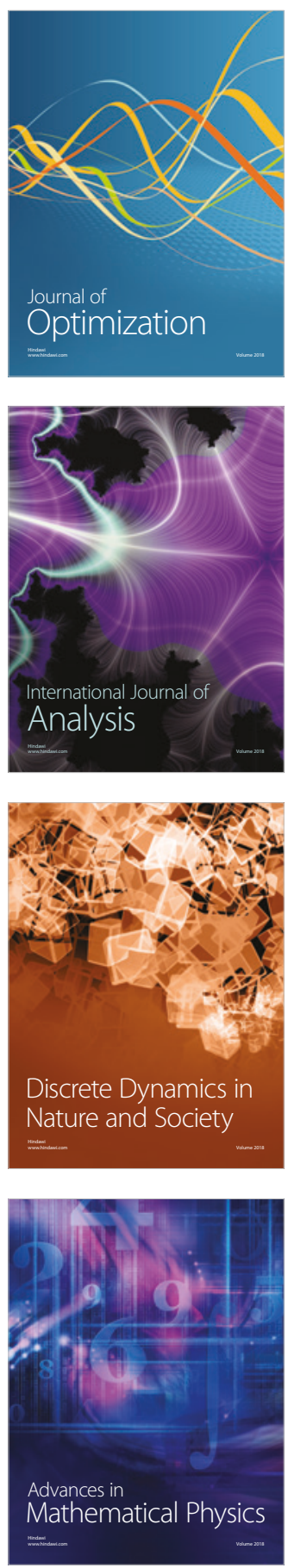